
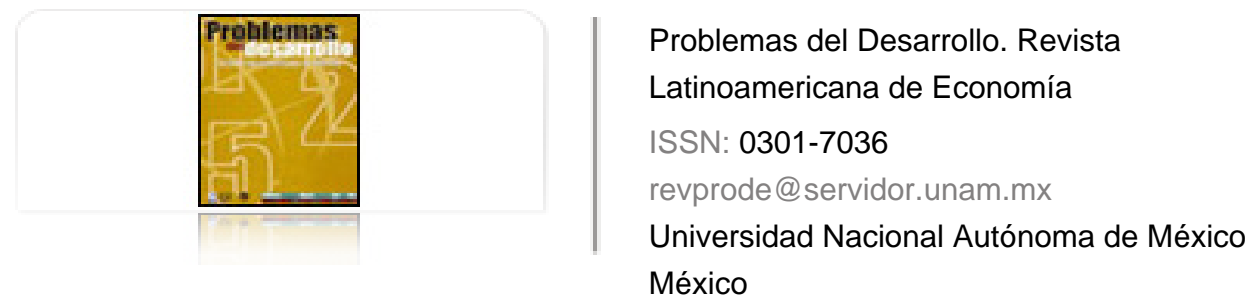

Escalante, Roberto; Catalán, Horacio

El sector de manufacturas en Guanajuato y su participación en el mercado nacional Problemas del Desarrollo. Revista Latinoamericana de Economía, vol. 42, núm. 165, abril-junio, 2011, pp. $87-112$

Universidad Nacional Autónoma de México

Distrito Federal, México

Disponible en: http://www.redalyc.org/articulo.oa?id=11819780005

- Cómo citar el artículo

- Número completo

- Más información del artículo

Página de la revista en redalyc.org

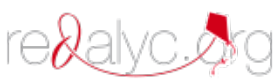

Sistema de Información Científica

Red de Revistas Científicas de América Latina, el Caribe, España y Portugal Proyecto académico sin fines de lucro, desarrollado bajo la iniciativa de acceso abierto 


\title{
El SECTOR DE MANUfaCturas EN Guanajuato Y SU PARTICIPACIÓN EN EL MERCADO NACIONAL
}

\author{
Roberto Escalante y Horacio Catalán*
}

Fecha de recepción: 15 de diciembre de 2010. Fecha de aceptación: 21 de marzo de 2011.

\begin{abstract}
RESUMEN
El objetivo del presente artículo es identificar los determinantes de la producción de la industria de manufacturas en Guanajuato en tres divisiones: alimentos, autopartes y calzado. Los resultados de las estimaciones econométricas muestran que la industria de autopartes es bastante sensible a las condiciones del mercado externo. La industria de alimentos está muy vinculada al mercado interno. La industria del calzado desde principios de la presente década ha enfrentado una crisis derivada de un crecimiento acelerado de las importaciones, y se espera que mantenga esta tendencia negativa en los próximos años. Condiciones favorables en el mercado externo promoverían un mayor crecimiento en las autopartes y el calzado, pero este resultado considera que las empresas tienen una capacidad de respuesta para adaptarse a las nuevas condiciones y mejorar su posicionamiento en el mercado nacional. Factores importantes a considerar en el diseño de una política industrial a nivel estatal.
\end{abstract} Palabras clave: manufacturas, mercado, producción.

\section{The MANUfaCtURING SECTOR IN GUANAJUTO AND ITS SHARE IN THE NATIONAL MARKET}

\begin{abstract}
The aim of this article is to identify the determinants of production in Guanajuato's manufacturing industry in three divisions: food, autoparts and footwear. The results of the econometric estimates show that the autoparts industry is quite sensitive to external market conditions. The food industry is strongly linked to the domestic market. The footwear industry, since the beginning of this decade has been facing a crisis derived from the rapid growth of imports, and it is expected to maintain this negative trend for the next few years. Favorable conditions in the external market drove the increased growth of autoparts and footwear, but this result assumes that companies are able to respond and to adapt themselves to the new conditions and improve their positioning in the national market-important factors to consider in designing an industrial policy at a state level.
\end{abstract}

Key words: manufacturing, market, production.

* Profesores de la Facultad de Economía, UnAM, correo electrónico: semerena@servidor.unam. mx, catalanh@economia.unam.mx. Agradecemos el apoyo en las estimaciones econométricas a Carlos Francisco Cruz. Desde luego se aplica el descargo usual de los errores. 


\section{LE SECTEUR DES MANUFACTURES DANS L'ÉTAT DE GUANAJUATO ET LEUR PARTICIPATION AU MARCHÉ NATIONAL (MEXICAIN) \\ Résumé}

L'objectif de cet article est d'identifier les facteurs de production de l'industrie des manufactures de l'état mexicain de Guanajuato dans trois secteurs : alimentation, pièces automobiles et chaussures. Les résultats des estimations économétriques montrent que l'industrie des pièces automobiles est assez sensible aux conditions du marché externe. L'industrie de l'alimentation est mieux reliée au marché interne. L'industrie de la chaussure a affronté, depuis le début de la décennie en cours, une crise découlant d'une croissance accélérée des importations, et il est prévu qu'elle maintienne cette tendance négative dans les prochaines années. Des conditions favorables sur le marché externe permettraient une croissance majeure dans les pièces automobiles et la chaussure, mais ce en considérant que les entreprises ont une capacité de réponse pour s'adapter aux nouvelles conditions et améliorer leur positionnement sur le marché national. Facteurs qu'il est important de considérer dans la détermination d'une politique industrielle au niveau de l'état.

Mots clés : manufactures, marché, production.

\section{O SETOR DE MANUFATURAS EM GUANAJUATO E SUA PARTICIPAÇÃO NO MERCADO NACIONAL}

\section{Resumo}

O objetivo do presente artigo é identificar os determinantes da produção da indústria de manufaturas em Guanajuato em três divisóes: alimentos auto-partes e calçado. Os resultados das estimaçóes econométricas mostram que a indústria de auto-partes é bastante sensível às condiçôes do mercado externo. A indústria de alimentos está muito vinculada ao mercado interno. A indústria do calçado desde princípios da presente década enfrentaram uma crise derivada de um crescimento acelerado das importaçóes, e se espera que mantenha esta tendência negativa nos próximos anos. Condiçóes favoráveis no mercado externo promoveriam um maior crescimento no setor de auto-partes e de calçado, mas este resultado considera que as empresas têm capacidade de resposta para se adaptar às novas condiçóes e melhorar o seu posicionamento no mercado nacional. Fatores importantes a considerar no desenho de uma política industrial a nível estatal.

Palavras-chave: Manufaturas, mercado, produção.

瓜纳华托州制造业及其在国内市场的参与

罗伯托・埃斯卡兰特及欧拉西奥・卡塔兰 概要

这篇文章旨在辨明决定瓜纳华托州制造业在食品、零部件和鞋业三个方面 的产量的因素。计量经济的估算结果表明零部件产业对外部市场条件相当 敏感。食品业则与内部市场关系紧密。鞋业自这个十年初以来就面临着由 进口加速而带来的危机, 而这种不利的趋势在接下来的几年里还将延续。 外部市场的有利条件将促进零部件产业和鞋业的更快增长, 但这里得出的 结论认为相关企业有能力适应新环境并取得更好的国内市场定位。这些是 制定国家级产业政策时应当考量的要素。

关键词: 制造业, 市场, 产量 


\section{INTRODUCCIÓN}

En las últimas tres décadas la economía mexicana ha enfrentado un proceso de transformación en su estructura productiva, que ha derivado en un cambio en la ubicación espacial de las actividades económicas, propiciando una especialización de ciertas regiones y estados. En este contexto, existen diversos factores que pueden explicar el desarrollo de ciertas regiones al interior del país (Fujita y Thisse, 2002; Black y Henderson, 1999), entre las que se destacan: factores económicos; el aumento natural de la población; el nivel de educación de la población o nivel de capital humano (Tamura, 1996); la migración a las ciudades; las ventajas originadas en las externalidades positivas de centros urbanos (Moomaw, 1981); los costos de transporte y de transacciones en actividades de servicios (Krugman, 1991); ubicación geográfica (Gallup, et al, 1999). En el caso particular de la producción de manufacturas se ha señalado que los costos de transporte y las economías de escala en la producción, adquieren una mayor ponderación en la decisión de las empresas para ubicar sus actividades en pequeñas zonas geográficas, como ciudades de tamaño medio (Rotemberg y Saloner, 2000).

Por otra parte, un aspecto que ha recibido una menor atención en los determinantes del desarrollo regional, es el comercio internacional y en específico la apertura comercial. Este proceso de apertura comercial y mayor competencia con el mercado internacional, genera una reasignación a nivel sectorial y regional de los recursos, modificando el patrón de especialización al interior de los países (Hanson, 1998). En el caso de la economía mexicana, desde finales de la década de los ochenta inició un proceso de apertura comercial que se amplió con la entrada en vigor del Tratado de Libre Comercio (TLC) en 1994. Las investigaciones empíricas sobre el impacto de la apertura comercial en las manufacturas han establecido "hechos estilizados" que sugieren que el TLC, conjuntamente con aspectos de la geografía económica tales como la estructura de mercado y los encadenamientos hacia atrás y hacia delante, se han convertido en factores que impactan a la estructura de la producción de varias ramas de las manufactureras y en particular a los estados del centro del país (Sargent y Matthews, 2001, Hanson, 1998, Davis y Weinstein, 1998, Mendoza, 2003).

Se aprecia que los efectos regionales al interior del país son superiores a los efectos internacionales, debido a que los costos de transporte y las barreras no arancelarias son más bajos entre regiones de países que entre países y, además, por la existencia de mayor movilidad de factores de la producción entre regiones que entre países (Mendoza, 2003). Sin embargo, este tipo de estudios no permite identificar los efectos que tiene la apertura comercial en la participación de la producción de manufacturas a nivel regional en el mercado doméstico y, 
en consecuencia, en el empleo y las posibilidades de expansión. Así, el objetivo principal del presente artículo es identificar, mediante modelos econométricos, los determinantes macroeconómicos de la producción de manufactureras en el Estado de Guanajuato, en tres divisiones relevantes para la entidad: alimentos, autopartes, piel y calzado. Estos modelos pueden utilizarse para realizar simulaciones del impacto de una mayor competencia del exterior así como un mayor estímulo a las exportaciones, a fin de medir su participación en el mercado interno. El artículo se divide en cuatro apartados, incluyendo a la presente introducción. El segundo apartado, muestra un análisis de las trayectorias de la producción de manufacturas en Guanajuato en años recientes. La tercera, presenta la evidencia empírica apoyada en los modelos econométricos y, finalmente, las conclusiones.

\section{EVOLUCIÓN DEL SECTOR DE MANUFACTURAS EN GUANAJUATO}

En las últimas dos décadas, la economía del Estado de Guanajuato ${ }^{1}$ ha mostrado un gran dinamismo en el crecimiento económico, por arriba del promedio nacional. Actualmente, la entidad contribuye con el 3.7\% del PIB nacional. La evolución reciente de la economía del estado se basa en el crecimiento de sectores como el de manufacturas, que contribuye con el $28 \%$ de la producción estatal, así como el comercio y los servicios. Al interior de la economía del estado (Gráfica 1), en los sectores con mayor contribución relativa se ubican los servicios con $35.5 \%$ del piB de la entidad. Este porcentaje se ha mantenido relativamente estable en la última década. Por su parte, el sector de las manufacturas que contribuye a la actividad económica con un $28 \%$, es el sector que muestra una participación bastante elevada con respecto al promedio nacional, el cual se ubica en 17\% (INEGI, 2009). De tal manera que el estado de Guanajuato registra un alto nivel de industrialización, así como un gran dinamismo del sector servicios, lo cual le permite contar con una base económica importante para ofrecer oportunidades de inversión. El comercio, también registra una contribución destacada en la economía de la entidad y contribuye con el $16.3 \%$ del ingreso de los habitantes del estado. Sin embargo, otros sectores como la construcción y las actividades agropecuarias, han perdido presencia en la actividad global y actualmente su contribución al ingreso es de $4.6 \%$ en el caso del sector agropecuario, ligeramente superior al promedio nacional, en tanto que la construcción aporta el $6.5 \%$, prácticamente igual al valor del promedio nacional (6.4\%).

\footnotetext{
1 Con base en información de INEGI
} 
Gráfica 1. PIB sectorial de la economía de Guanajuato 2003-2008

(porcentaje)

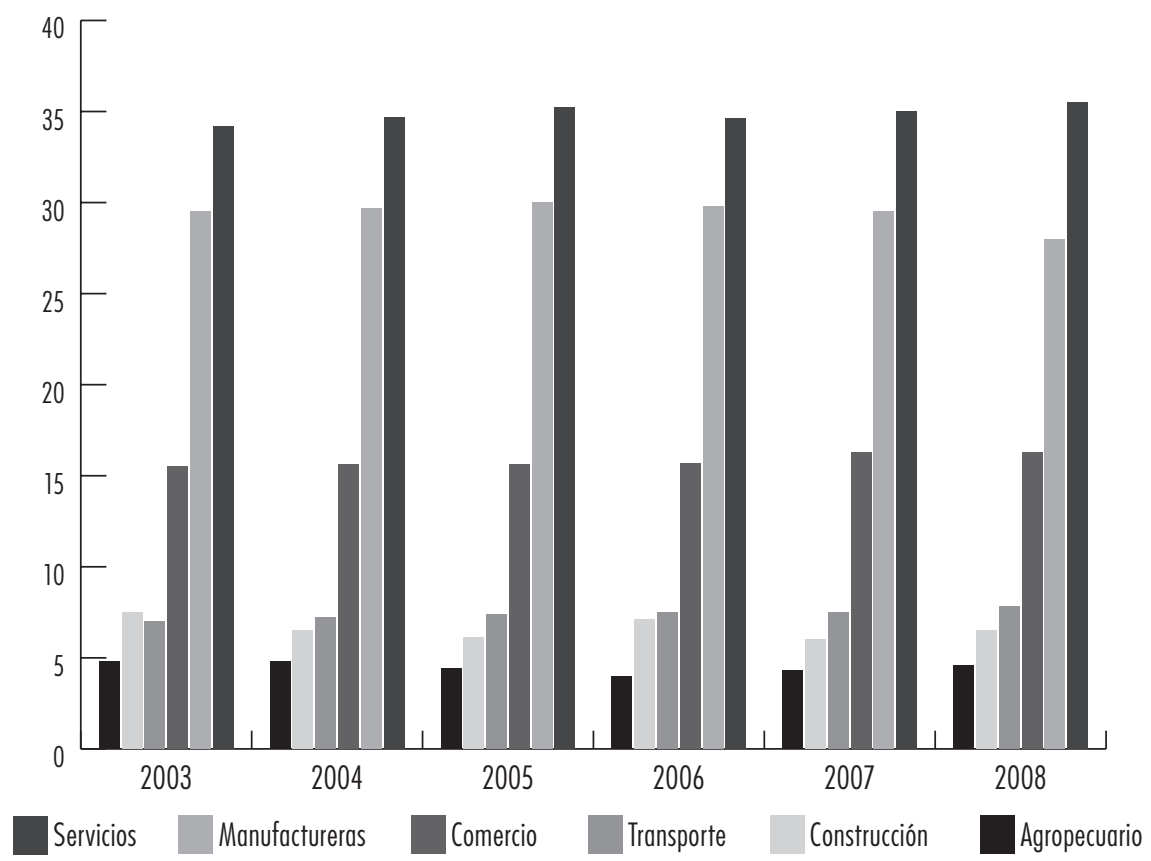

Fuente: Con base en información de INEGI.

Es importante señalar que actualmente la entidad ocupa el sexto lugar, en términos de su contribución al PIB de manufacturas a nivel nacional, ubicándose en el grupo de las entidades con mayor importancia económica en México, por debajo de las entidades más importantes del país como el Estado de México, Distrito Federal, Jalisco y Nuevo León (Gráfica 2). Asimismo, en términos del empleo, también concentra el $6 \%$ de los puestos de trabajo del sector manufacturas a nivel nacional. Es importante destacar que en el periodo de 1993 a 2008 el conjunto de las actividades de las manufacturas en el estado de Guanajuato reportaron un crecimiento promedio anual de 7\%, superior al promedio nacional de 4\% anual, para el mismo periodo de referencia (INEGI, 2009) Es decir, las empresas instaladas en la entidad muestran un mayor dinamismo, indicando que el estado de Guanajuato en algún sentido cuenta con las condiciones favorables para el desarrollo de este tipo de empresas. 
Gráfica 2. Principales entidades que contribuyen al PIB de manufacturas a nivel nacional 2008 (porcentaje)

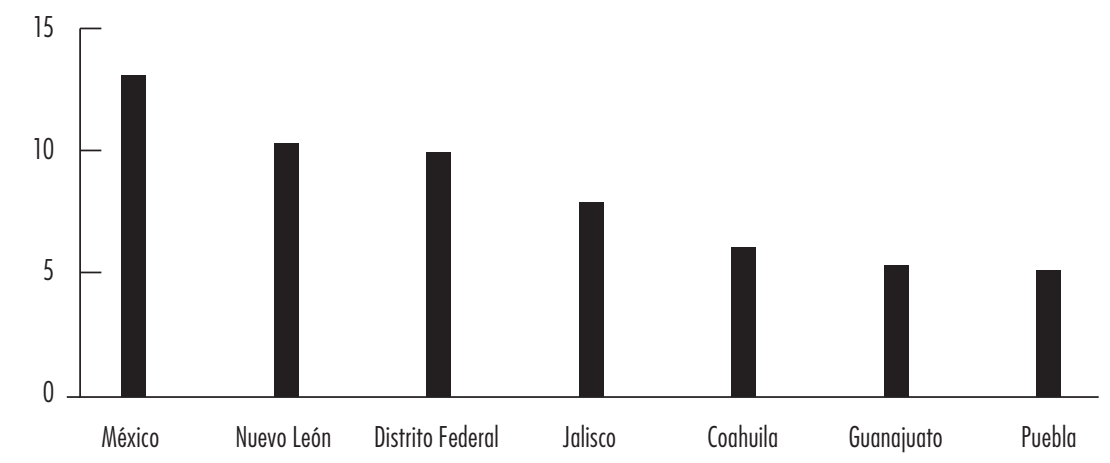

Fuente: Con base en información de INEGI.

No obstante que no se dispone de información histórica para el estado de Guanajuato, en términos de las actividades industriales, es posible estimar una serie de tiempo para el PIB de manufacturas desde mediados de la década de los ochenta a la actualidad. ${ }^{2}$ En la Gráfica 3 se aprecia que la producción manufacturera en Guanajuato, desde mediados de la década de los ochenta, había registrado un continuo crecimiento, el cual se acelera a partir de 1995, ańo en el cual se inicia un rompimiento de la tendencia de largo plazo hacia un mayor nivel de producción. Así, por ejemplo, en el periodo de 1985 a 1993, el PIB de manufacturas de la entidad creció a un ritmo de 3.8\% anual, un punto porcentual por arriba del promedio nacional. Con el inicio del Tratado de Libre Comercio entre Estados Unidos, Canadá y México, la actividad industrial en la entidad recibió un fuerte impulso. En el periodo de 1995 a 2002, a nivel nacional, la producción de manufacturas se recuperó y creció en promedio anual 4.7\%. En contraste, estas actividades en Guanajuato registraron un elevado ritmo de crecimiento de $9.6 \%$ anual para el mismo periodo de referencia (INEGI, 2009).

Este dinamismo del sector en la entidad ha permitido que su contribución a nivel nacional muestre una clara tendencia ascendente, pasando de niveles de 3.2

2 En este caso se utiliza información histórica de INEGI sobre el PIB estatal por gran división, y considerando la participación porcentual en el estado y por medio de métodos de interpolación es factible obtener una tendencia de largo plazo del рів de manufacturas para el estado de Guanajuato. 
Gráfica 3. Indicadores del PIB de manufacturas en Guanajuato
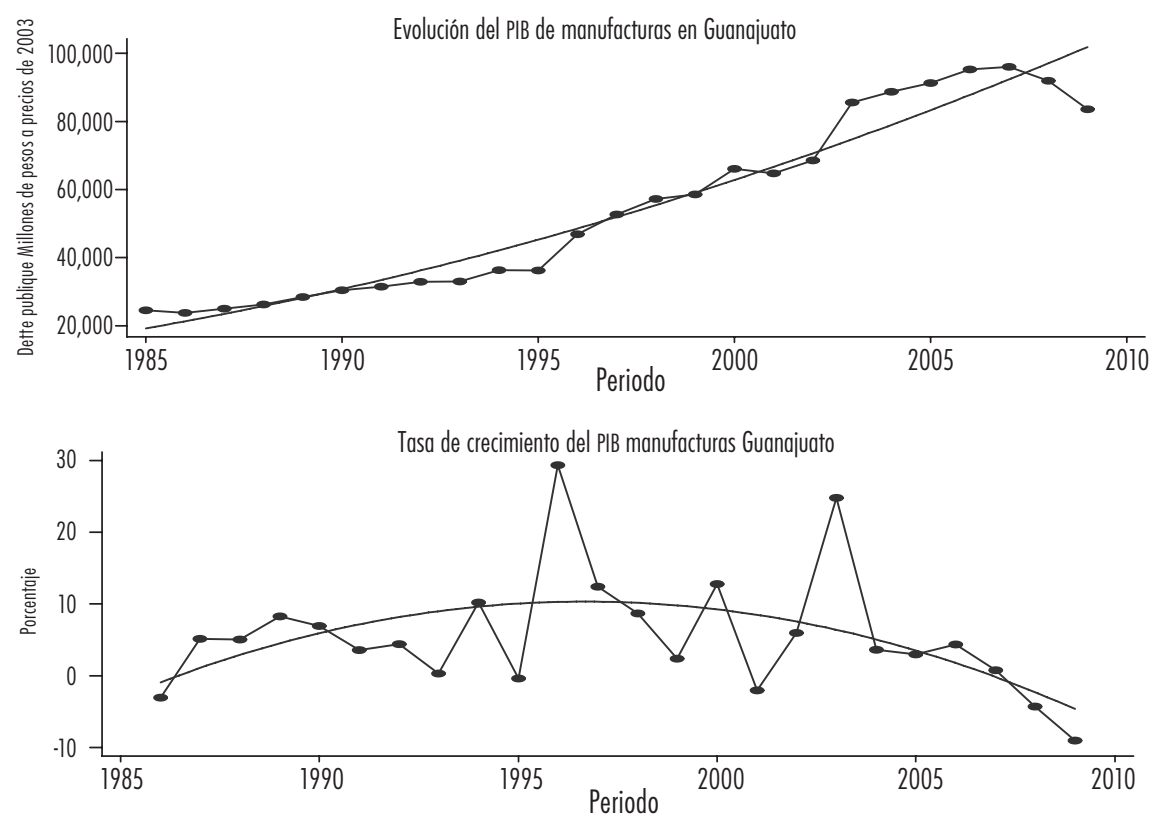

Participación de Guanajuato en el PIB de manufacturas nacional
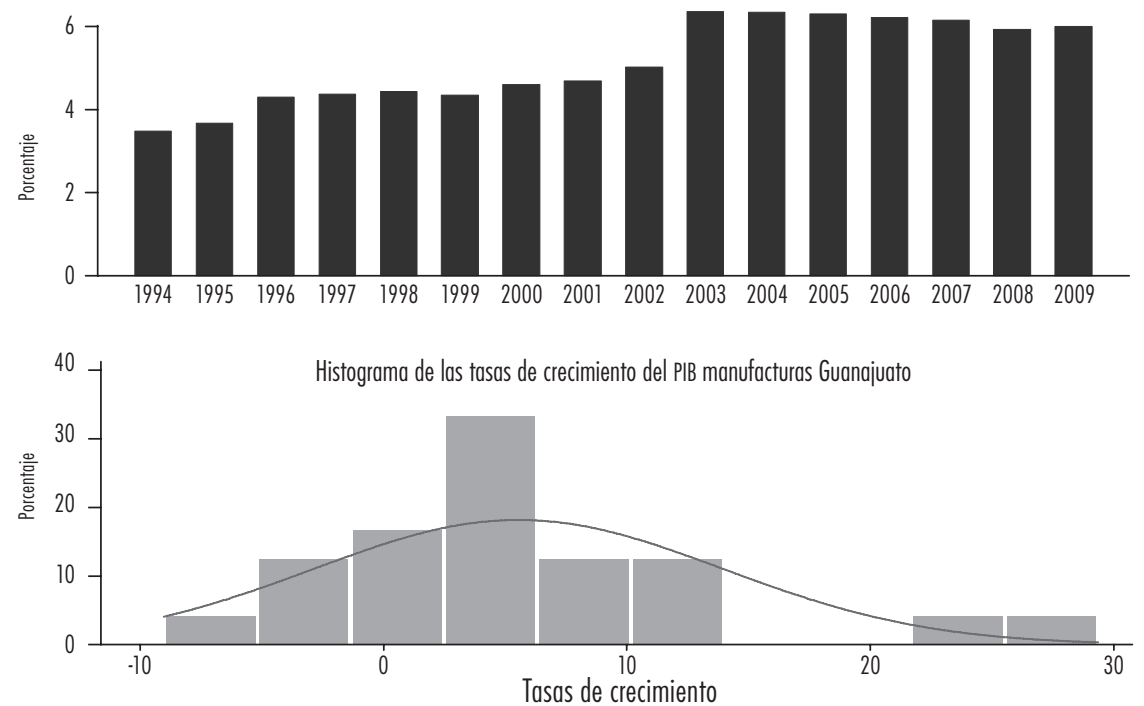

Fuente: Con base en información de INEGI. 
puntos porcentuales en 1992 a 5 puntos porcentuales en 2002. Actualmente, su contribución se ubica en 6 puntos del PIB de manufacturas a nivel nacional, indicando la gran importancia del estado a nivel nacional, y también una mayor especialización de la entidad en la producción de manufacturas. En efecto, el índice de especialización de manufacturas en la entidad se ubicaba en un valor de 0.97 en 1993. En 2004, el valor de este indicador fue de 1.4 y actualmente se reporta un valor de $1.53 .^{3}$

Distintos factores han influido en el desempeño de las manufacturas en Guanajuato. Sin embargo, este patrón también se registra en otras entidades del país. Las investigaciones empíricas sobre el tema han establecido "hechos estilizados" que sugieren que la apertura comercial, sobre todo a partir del TLC, conjuntamente con aspectos de la geografía económica tales como: la estructura de mercado y los encadenamientos hacia atrás y hacia delante, se han convertido en factores que impactan a la estructura de la producción de varias industrias manufactureras al interior del país (Davis y Weinstein, 1998, Mendoza, 2003). En particular, se aprecia que los efectos regionales al interior del país son superiores a los efectos internacionales, debido a que los costos de transporte y las barreras no arancelarias son más bajos entre regiones de países que entre países (Mendoza, 2003).

Así, por ejemplo, en la investigación de Mendoza (2003), por medio de un modelo econométrico concluye que la apertura comercial fue un elemento clave en la decisión de la localización de la industria manufacturera en los estados del centro norte del país, tanto por su vinculación con el sector exportador y por el crecimiento del mercado interno. De tal manera que la razón por la cual la industria no se localizó con mayor intensidad en la frontera norte se debe a que la región central mantiene un liderazgo en términos de mercado interno. En particular, los costos negativos generados a partir de la excesiva concentración

3 Este índice ubica la especialización estatal, en función del tamaño del sector económico en la entidad, aproximado por el PIB de la división en el PIB estatal, respecto al peso del mismo sector en el conjunto de la economía, es decir el PIв de la división en el pIB nacional. De tal manera que si el valor del coeficiente es igual a 1 significa que el tamaño de ese sector, en la entidad, es idéntico al tamaño del sector en el país y, por tanto, no hay especialización. Si su valor es menor que 1 , se infiere que el sector tiene un tamaño proporcionalmente menor en el estado, que el mismo indicador en el país y, por tanto, no hay especialización. Un coeficiente mayor a la unidad, significa que el peso de ese sector en el estado es proporcionalmente mayor que el peso que tiene en la economía nacional y, por tanto, la entidad está especializada en esa actividad. 
y congestión urbana, previos a la apertura, provocaron que la industria buscara situarse en una región central alterna a la Ciudad de México y el Estado de México, siendo los estados centrales contiguos la mejor alternativa.

No obstante el impulso a la industria de manufacturas, en los últimos 4 años se ha perdido dinamismo. En efecto, considerando el periodo de 2006 a 2009, se registra una contracción promedio de $4.3 \%$ anual (INEGI, 2009). Como se observa en la Gráfica 3, las tasas de crecimiento de la producción de manufacturas en Guanajuato se han ubicado en una fase de claro descenso. Si bien a nivel nacional las manufacturas están en una fase de contracción, en el caso de la entidad se ha ubicado por debajo del promedio nacional. Es decir, dada la orientación de la producción tanto al mercado de exportación como al mercado interno, la entidad ha resentido en mayor medida el impacto de la recesión a nivel mundial como la fuerte contracción del mercado interno, lo cual muestra evidencia de que las empresas ubicadas en el estado de Guanajuato, presentan una mayor asociación al ciclo de la economía internacional y al mercado interno, por lo tanto existen diversos factores que influyen en su comportamiento a corto plazo. En este contexto, resulta relevante contar con indicadores sobre la evolución del mercado, así como una constante revisión de escenarios prospectivos a corto y mediano plazo. Los subsectores de gran relevancia para el estado, por su impacto en el empleo y su contribución a nivel nacional son: industria de autopartes, industria de alimentos e industria del calzado. El objetivo es especificar y estimar modelos econométricos de estas industrias que sirvan de base para realizar análisis prospectivo sobre la participación de mercado, mediante la evaluación de distintos escenarios alternativos.

\section{METODOLOGÍA ECONOMÉTRICA Y EVIDENCIA EMPÍRICA}

El análisis se centra en identificar la participación en el mercado de cada una de las ramas de las manufacturas del estado de Guanajuato en el mercado nacional, en función de un conjunto de variables que miden las características del mercado así como la competencia externa. Los modelos se basan en una ecuación de demanda tradicional ${ }^{4}$ (Deaton y Muellbauer, 1980) incluyendo al ingreso, ${ }^{5}$ a los precios relativos del propio bien y, adicionalmente, se incluyen los

4 El análisis de la demanda se fundamenta a partir de la teórica económica convencional (anexo 1).

5 En algunos casos se utiliza el producto per capita o la estructura poblacional como variable explicativa en la medida en que la demanda de algunos bienes depende de la edad. 
impactos de la competencia externa por medio del nivel de importaciones, las condiciones del mercado externo a través del nivel de exportaciones e importaciones en la industria. Así, la especificación general del modelo se basa en la siguiente ecuación.

$$
q_{t}=\beta_{0}+\beta_{1} y_{t}+\beta_{2}\left[p_{i} p_{t}\right]+\beta_{3} m_{t}+\beta_{4} x_{t}+u_{t}
$$

Donde $q_{t}$ representa el valor de producción de la rama de producción alimentos $\left(q_{a l i}\right)$ autopartes $\left(q a u t_{t}\right)$ y piel y calzado $(q p y c) ; y_{t}$ el ingreso real que representa al mercado doméstico; $\left[p_{i} p_{t}\right]$ el precio relativo del conjunto de bienes que se produce en la rama de referencia; $m_{t}$ es el nivel de importaciones de los bienes que produce la industria como proporción del mercado interno, que aproxima el nivel de competencia en el mercado interno y $x_{t}$ es la proporción de exportaciones sobre el valor de las ventas totales que miden la capacidad de ventas al exterior de la industria. Las letras en minúsculas representan el logaritmo natural de las series.

Las estimaciones de la ecuación (1) para las tres divisiones de manufacturas para el estado de Guanajuato se basaron en el uso de métodos econométricos que consideran el orden de integración de las series y la posible presencia del problema de regresión espuria ${ }^{6}$ (Granger y Newbold, 1974, Enders, 1995). La información estadística corresponde a series mensuales del periodo de 2005(1) a 2009(12). Se considera el valor de la producción de cada industria en miles de pesos a precios de 2003, como una medida del mercado doméstico, en frecuencia mensual, se utiliza el Índice Global de la Actividad Económica (IGAE). Los precios relativos se definen como la relación entre los índices de precios al consumidor de cada industria en relación al índice nacional de precios al consumidor, ambos base año 2003. El nivel de importaciones se aproxima como el coeficiente de las importaciones a nivel nacional de toda la industria sobre el Consumo Nacional Aparente de toda la industria, y el nivel de exportaciones se estima como la proporción de las exportaciones de toda la industria a nivel nacional sobre el valor total de las ventas. Estas variables permiten aproximar el nivel de competencia del exterior en el mercado nacional que afecta negativamente a la industria doméstica, así como la expansión del mercado externo que tiene un impacto positivo.

En primera instancia se identificó el orden de integración de las distintas variables a través de aplicar las pruebas de raíz unitaria ${ }^{7}$ Dickey Fuller Aumentada

\footnotetext{
$6 \quad$ En anexo se expone la metodología econométrica.

7 La pruebas de raíz unitaria se realizaron en el Software RATs.
} 
(ADF) (1981), de Phillips Perron (PP) (1988) y KPSs (Kwiatkowsky, et al, 1992). Este se reporta en el Cuadro A1 del anexo. ${ }^{8}$ Es importante señalar que las pruebas ADF y PP utilizan como hipótesis nula el que la serie tiene raíz unitaria a diferencia de la prueba KPSs, que tiene como hipótesis nula el que la serie es estacionaria lo que busca minimizar la posibilidad de realizar inferencias estadísticas equivocadas (Maddala y Kim, 1998).

Los resultados muestran que las tres series que miden el valor bruto de la producción de las industrias de alimentos, autopartes y calzado, pueden considerarse como series estacionarias alrededor de tendencia determinística, por lo cual no muestran una trayectoria estable en el tiempo. Además, los componentes tendencia y constante en la serie es evidencia indirecta de la presencia de cambios estructurales en las series. Las series que miden los precios relativos de las divisiones respectivas, en principio, deberían resultar series estacionarias. Sin embargo, las pruebas indican que son series de orden de integración I(1) y en todos los casos los componentes de tendencia o constante, no aportan información para caracterizar el proceso estocástico de las series. Se pueden considerar como de orden de integración I(1), es decir describen un proceso estocástico de camino aleatorio. Las variables que miden el nivel de competencia externa por medio de las importaciones, también se pueden considerar como no estacionarias. Un resultado similar se presenta en la proporción de exportaciones respecto a las ventas totales. Las pruebas de raíz unitaria concluyen que son series de orden de integración I(1).

Se incluyen dos variables más que miden la actividad económica como es el caso del Indicador Global de la Actividad Económica (IGAE), denotada como $y_{t}$, que aproxima la dinámica del mercado doméstico. Las pruebas de raíz unitaria concluyen que esta serie se puede considerar como de orden de integración I(1), es decir describe un proceso estocástico no estacionario. Estos resultados muestran que las series consideradas presentan propiedades estocásticas diferentes que deben ser consideradas en la modelación econométrica. En este sentido, la mejor opción es, por tanto, especificar un modelo de corrección de errores,

8 La prueba ADF se basó en el procedimiento "de lo general a lo específico" a través de estimar en principio regresiones con constante y tendencia, verificando su significancia estadística. El número de rezagos $(\mathrm{k})$ fue determinado mediante el criterio de significancia estadística de la prueba t, procedimiento que es conocido como t-sig ( $\mathrm{Ng}$ y Perron, 1995). Por su parte, las pruebas PP y KPSS, utilizan una corrección semiparamétrica de la varianza de los errores de la prueba que depende del tamaño de la muestra (Phillips y Perron, 1988). 
incluyendo las desviaciones de la serie respecto al vector de cointegración. Por lo tanto, es necesario estimar una ecuación de largo plazo entre el conjunto de variables, por medio del método de Johansen (1988).

Con base en estos resultados se aplicó el procedimiento de Johansen (1988 y 1995) para el valor bruto de producción de las tres divisiones de las manufacturas: industria de alimentos ( $q$ al $l_{t}$, valor bruto de la producción de autopartes $\left(q a p_{t}\right)$ y valor bruto de la producción de piel y calzado $(q c a l)$. En principio, se procedió a especificar un modelo var para cada una de las divisiones, cuyo número de rezagos fue seleccionado a partir de los criterios de información estadística. Por otra parte, a fin de considerar el problema de cambio estructural en el espacio de cointegración, se incluyeron variables "dummy" de pulso en cada uno de los moldeos VAR. Los resultados de la prueba de la traza para determinar el número de vectores de cointegración se reportan en el Cuadro 1.

Cuadro 1. Resultados de la pruebas de la Traza del Procedimiento de Johansen

\begin{tabular}{ccccc}
\hline$H_{0}$ & Alimentos & Autopartes & Piel y calzado & $\begin{array}{c}\text { Valor crítico } \\
(95 \%)\end{array}$ \\
\hline$r=0$ & $169.48^{\star}$ & $113.2^{\star \star}$ & $246.5^{\star \star}$ & 76.97 \\
$r \leq 1$ & $99.40^{\star}$ & $60.09^{\star \star}$ & $135.9^{\star \star}$ & 54.07 \\
$r \leq 2$ & $57.75^{\star}$ & 24.27 & $79.64^{\star \star}$ & 35.19 \\
$r \leq 3$ & 16.93 & 9.35 & 17.8 & 20.00 \\
$r \leq 4$ & 2.32 & 3.71 & 8.26 & 9.20 \\
\hline
\end{tabular}

Notas: $\left.{ }^{\star}\right)$ Indica rechazo de la hipótesis nula. La hipótesis nula es aceptada cuando el valor calculado del estadístico de la Traza es menor al valor crítico.

Alimentos: VAR con 2 rezagos, dummy febrero 2009.

Autopartes: VAR con 2 rezagos, dummy diciembre de 2008 y septiembre de 2007.

Piel y calzado: Var con 1 rezago, dummy enero de 2007.

Periodo: 2005(1)-2009(12).

En el caso de las industrias de alimentos así como de piel y calzado, indica que pueden existir hasta 3 vectores de cointegración, y la industria de autopartes la prueba de la traza reporta la presencia de al menos dos vectores. Es decir en las tres industrias se presenta un problema de múltiple cointegración (Harldrup, 1998). Una explicación de este resultado, es que algunas variables en el modelo var pueden ser de orden de integración I(2) y en consecuencia existe un vector de cointegración en el espacio I(1), que tendría que probarse en el marco del procedimiento de Johansen (1995) y, por lo tanto, alguna de las variables, 
debería incluirse en la relación de largo plazo en primera diferencia. Por otra parte, indicaría que existe más de una relación de equilibrio entre el conjunto de variables, pero esto implica identificar los distintos vectores de cointegración (Patterson, 2000 y Juselius, 2006), por lo cual se recomienda imponer restricciones en los vectores de cointegración a fin de identificar sus parámetros y definir las distintas ecuaciones.

Las pruebas de raíz unitaria no señalan que alguna de las variables sea de orden de integración I(2). Por lo tanto, podemos descartar soluciones en el espacio I(2). La identificación de los vectores de cointegración implica asumir una hipótesis teórica sobre la relación de las variables que sea consistente, es decir que existe un modelo general de soluciones simultáneas entre las variables explicativas. En este sentido, se debería justificar teóricamente una ecuación. Por ejemplo, para los precios relativos o los coeficientes de importaciones y exportaciones en función del resto de las variables, lo cual no se justifica desde el ámbito de la teoría económica. Así, se consideró el primer vector de cointegración y normalizando con respecto a la primera variable, se define una ecuación del valor de la producción. Los vectores de cointegración normalizados se presentan en el Cuadro 2. Los resultados en las tres ecuaciones son consistentes con la hipótesis teórica inicial, el mercado doméstico aproximado por el IGAE muestra una elasticidad positiva, los precios relativos mantienen una relación negativa con el valor de la producción así como una mayor competencia externa, en tanto que una promoción hacia el mercado externo reporta una elasticidad positiva respecto al valor de la producción.

Cuadro 2. Vectores de cointegración normalizados

\begin{tabular}{lccccc}
\hline Ecuación & $C$ & $y_{t}$ & $p r_{t}$ & $m_{t}$ & $x_{t}$ \\
\hline Alimentos & 9.933 & 0.900 & -0.028 & -1.283 & 0.686 \\
Autopartes & 2.344 & 0.965 & -1.093 & -0.039 & 0.297 \\
Calzado y piel & 10.66 & 1.221 & -0.529 & -0.189 & 0.123 \\
\hline
\end{tabular}

Las condiciones del mercado interno tienen un mayor impacto en la producción de la industria de cuero y calzado, debido a que su elasticidad es superior a la unidad (1.2). Así, por ejemplo, si la actividad económica del país creciera a una tasa del $5 \%$ la producción de piel y calzado, en el estado de Guanajuato aumentaría en $6.1 \%$, es decir la respuesta de la producción es más que proporcional. La producción de la industria de autopartes también es altamente sensible a las condiciones del mercado interno, con una elasticidad del orden de 0.96. Así, 
considerando el mismo supuesto sobre el crecimiento de la economía, el sector reportaría una tasa de $4.8 \%$, en tanto que la industria de alimentos reportaría un crecimiento similar (4.5\%). Los resultados en las tasas de crecimiento asumen que el resto de los factores no se modifican.

La elasticidad precio en las tres industrias es negativa, que es consistente con la hipótesis teórica inicial, pero su magnitud es distinta para cada industria. En el caso de la industria de autopartes la elasticidad es igual a la unidad, por lo tanto un cambio en los precios de autopartes superior al incremento en el promedio general de los precios, implica una disminución en el valor de la producción en la misma proporción que el cambio en los precios. Calzado y piel muestran cierta sensibilidad al cambio en los precios relativos con una elasticidad de -0.52 , valor menor a la unidad. Por tanto, la producción de piel y calzado son menos sensibles a los cambios en los precios. Las empresas ubicadas en la producción de alimento en el estado de Guanajuato no muestran una sensibilidad respecto a los precios relativos. El valor de la elasticidad es muy cercano a cero.

En el caso de la competencia externa, la industria de alimentos es altamente sensible a las importaciones. El valor de la elasticidad es superior a la unidad $(-1.28)$, por lo que un aumento en las importaciones implicaría una diminución en el valor de la producción más que proporcional. La industria de piel y calzado también reportan un efecto negativo de las importaciones, pero en una menor magnitud (-0.18). Sin embargo, en la rama de autopartes, la elasticidad de importaciones no tiene un impacto importante en la producción doméstica. En la elasticidad de exportaciones para toda la industria, es decir una expansión del mercado externo influye, en mayor medida, en la producción de alimentos, y en una menor magnitud en autopartes y en piel y calzado.

La identificación de una relación de cointegración permite especificar un modelo de corrección de errores (Engel y Granger, 1987), que incluye las variables del vector de cointegración en primeras diferencia y las deviaciones de la variable dependiente respecto a su relación de largo plazo. Se estimaron, tres modelos de corrección y los resultados se presentan en las ecuaciones (2), (3) y (4):

\section{Industria de alimentos en Guanajuato}

(2) $\mathrm{Dqali}_{\mathrm{t}}=-0.315 \mathrm{Dqali}_{\mathrm{t}-4}+0.895 \mathrm{Dpr}_{\mathrm{t}-1}-1.586 \mathrm{Dpr}_{\mathrm{t}-2}+0.786 * \mathrm{Dy}_{\mathrm{t}}-0.786 * \mathrm{Dy}_{\mathrm{t}-2}$ $\begin{array}{llllll}\mathrm{t} \text {-estadístico } & (-2.890) & (1.193) & (-2.067) & (4.295) & (-1.571)\end{array}$ $\begin{array}{ccccc}-0.151 \mathrm{Dm}_{\mathrm{t}-3}+0.156 \mathrm{Dx}_{\mathrm{t}-2}-0.034 \mathrm{ecm}_{\mathrm{t}-1}-0.163 * \mathrm{~d} 0706 & -0.163 * \mathrm{~d} 0606 \\ \text { t-estadístico } & (-2.309) & (3.208) & (-1.341) & (-3.549)\end{array}$ 
Pruebas de diagnóstico:

$\mathrm{R}^{2}=0.594$

Normalidad J-B: 0.038 [0.980]

Autocorrelación LM(12): F = 1.756 [0.098]

Heterocedasticidad ARCH(12): $\mathrm{F}=1.653$ [0.123]

Error Cuadrático Medio: 0.041

Periodo: 2005(1) - 2009(12)

\section{Industria de autopartes en Guanajuato}

(3)

t-estadístico

$$
\begin{aligned}
& \text { Dqaut }_{t}=1.47+1.196 \mathrm{Dy}_{\mathrm{t}}-0.791 \mathrm{Dy}_{\mathrm{t}-1}-1.319 \mathrm{Dpr}_{\mathrm{t}} \\
& \begin{array}{c}
\text { (2.17) }(3.21) \\
(-2.06)
\end{array}
\end{aligned}
$$

$-0.229 \mathrm{Dm}_{\mathrm{t}}+0.198 \mathrm{Dx} \mathrm{t}_{\mathrm{t}-8}-0.158 \mathrm{ecm} \mathrm{t}_{-1}+0.218 \mathrm{~d} 0812-0.193 \mathrm{~d} 0912$

$\begin{array}{llllll}\mathrm{t} \text {-estadístico } & (-4.802) & (2.046) & (-2.164) & (4.864) & (-2.198)\end{array}$

Pruebas de diagnóstico:

$\mathrm{R}^{2}: 0.673$

Normalidad J-B: $0.663[0.717]$

Autocorrelación LM (12): $\mathrm{F}=0.915$ [0.543]

Heterocedasticidad ARCH (12): F $=2.415$ [0.864]

Error Cuadrático Medio: 0.077

Periodo: 2005 - 2009

\section{Industria de piel y calzado en Guanajuato}

(4) $\mathrm{Dqpyc}_{\mathrm{t}}=-0.140 \mathrm{Dqpyc}_{\mathrm{t}}+1.763 \mathrm{Dy}_{\mathrm{t}}-1.188 \mathrm{Dy}_{\mathrm{t}-1}-2.165 \mathrm{Dpr}_{\mathrm{t}-8}+1.571 \mathrm{Dpr}_{\mathrm{t}-12}$ $\begin{array}{lllll}\text { t-estad } & (-1.975) & (5.305) & (-3.462) & (-2.170)\end{array}$

$-0.352 \mathrm{Dm}_{\mathrm{t}-3}+0.106 \mathrm{Dx}_{\mathrm{t}-3}-0.355^{*} \mathrm{ecm}_{\mathrm{t}-1}+0.157^{*} \mathrm{D} 0812$

t-estad (-5.580) (2.017) (-2.934) (7.408)

Pruebas de diagnóstico:

$\mathrm{R}^{2}: 0.836$

Normalidad J-B: 0.419 [0.810]

Autocorrelación LM (12 rezagos): $\mathrm{F}=1.252[0.302]$

Heterocedasticidad ARCH (12 rezagos): $\mathrm{F}=1.735$ [0.126]

Error Cuadrático Medio: 0.064

Periodo: 2005-2009 
Las pruebas de especificación indican que los errores del modelo final no presentan problemas de autocorrelación, heterosedasticidad y se distribuyen como una función de densidad de probabilidad normal, es decir no contienen información sistemática que deba ser incorporada en el modelo o que afecte la estimación de los parámetros. Además, se observa que los valores proyectados por el modelo representan satisfactoriamente a los valores observados de las variaciones en el valor bruto de la producción de cada industria (Gráfica 4). En tanto que el mecanismo de corrección de errores es negativo y estadísticamente significativo, así que los desajustes en la relación de equilibrio no son permanentes y aportan información relevante para predecir las variaciones del valor bruto de la producción de las distintas industrias. Por lo tanto, los modelos de corto plazo pueden ser utilizados para realizar pronósticos y simulaciones.

A fin de evaluar el impacto de un cambio en algunas de las variables relevantes como una mayor competencia del exterior o un aumento de la demanda externa, se construye un primer escenario en el cual estas variables permanecen sin cambios, lo cual permite definir una trayectoria tendencial o escenario base (Business as Usual-BAU), del valor de la producción y, por tanto, de la participación en el mercado nacional, de las industrias de manufacturas en Guanajuato. El escenario base (Esc Base) define los siguientes supuestos:

Tasa de crecimiento del PIB Nacional de 4.1\% para 2010, 4.5\% para 2011 y $4.0 \%$ para 2012.

- Tasa de crecimiento de los Precios Relativos contante.

- Tasa de crecimiento de las Importaciones constante.

- Tasa de crecimiento de las Exportaciones constante.

En términos generales, el escenario base describe una trayectoria de la producción si las condiciones actuales no se modifican y si se logra una recuperación del crecimiento de la economía nacional. Esta trayectoria base es comparada con los datos preliminares observados en 2010 sobre la participación en el mercado de manufacturas de la entidad en el total nacional. Asimismo, con el propósito de evaluar el impacto de una mayor competencia internacional, se consideraron dos escenarios alternativos, que al generar nuevas trayectorias del valor de la producción se pueden comparar respecto al escenario base. Así tenemos el primer escenario alternativo, el cual asume un aumento de las importaciones (Escenario 1) en 3 puntos porcentuales de la producción nacional de 2010 a 2012, para las tres divisiones. El segundo escenario (Escenario 2), asume que existe una fuerte promoción a las exportaciones y en el mercado internacional se abren posibilidades de venta, así las exportaciones a nivel nacional crecen en 3 puntos porcentuales respecto a la producción nacional de 2010 a 2012. Estos valores se toman 
Gráfica 4. Valores observados y estimados de las variaciones del valor bruto de la producción (escala logarítmica de las series en primeras diferencias)
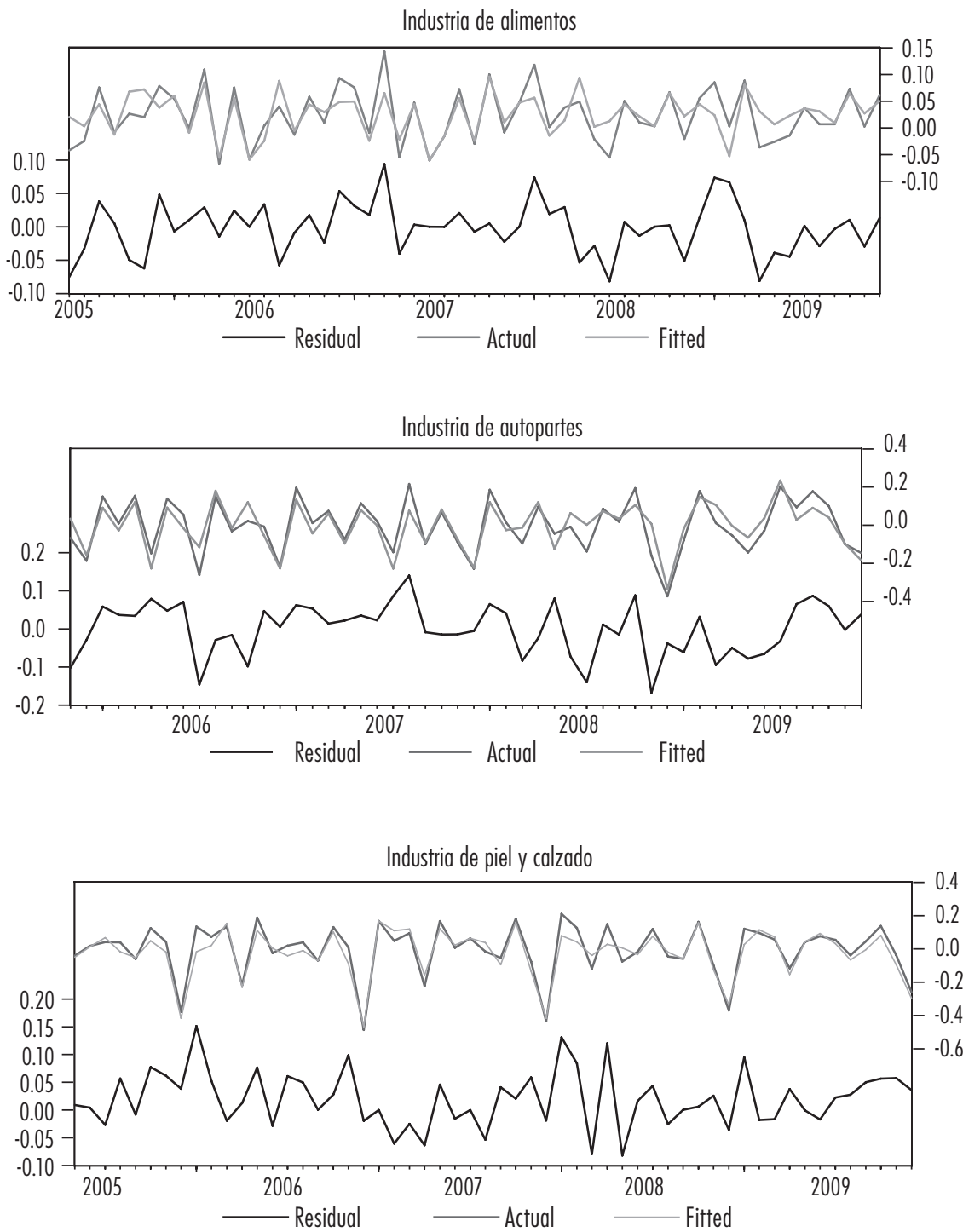

con base en las tasas históricas observadas. Los resultados de la simulación se presentan en las Gráficas 5, 6 y 7, para las divisiones de alimentos, autopartes, piel y calzado, respectivamente. 
Roberto Escalante y Horacio Catalán

Gráfica 5. Resultados para la industria de alimentos en Guanajuato

Alimentos: participación en el mercado nacional de la industria de Guanajuato

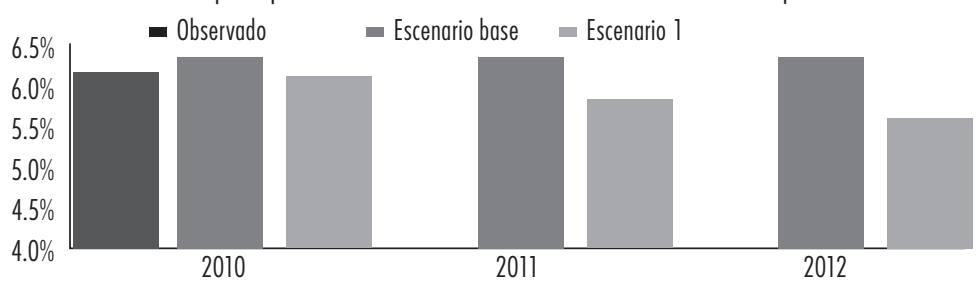

Alimentos: cambio en la participación del mercado a nivel nacional. Escenario 1 (puntos porcentuales)
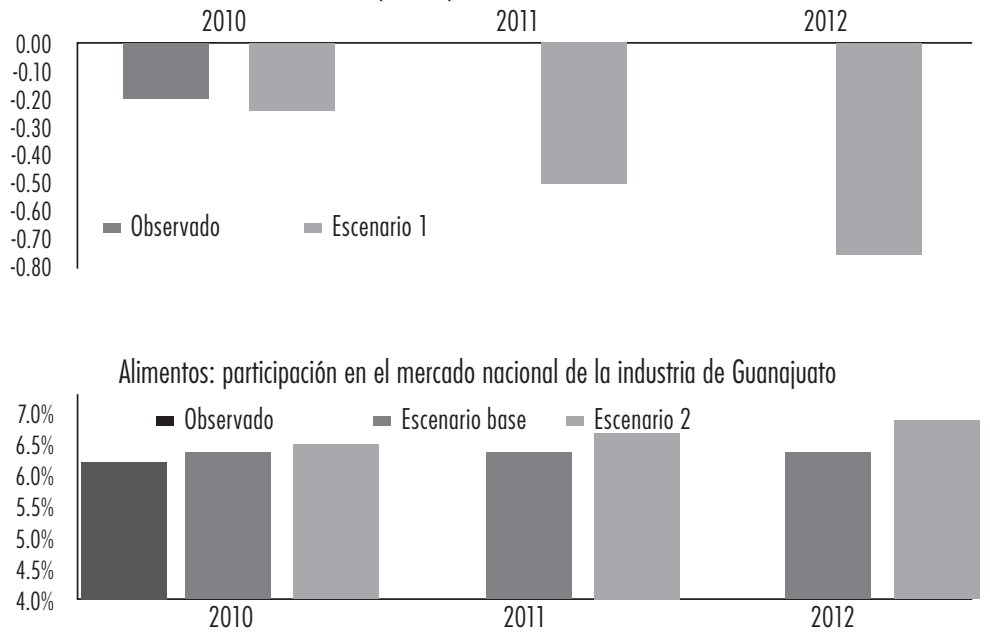

Alimentos: cambio en la participación del mercado a nivel nacional. Escenario 2 (puntos porcentuales)

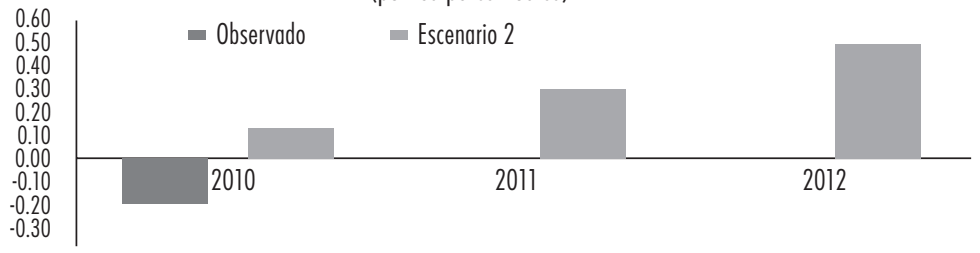

En el escenario base, si las condiciones actuales no cambian y el conjunto de la economía se recupera en los próximos tres años, la industria de alimentos en Guanajuato presentaría un crecimiento promedio de $2.3 \%$ anual, resultado muy similar al dato observado en 2010. Ante una expansión de las 
importaciones (Escenario 1), asociado a una mayor competencia del exterior, el impacto esperado en la producción de alimentos es una contracción de 1.9\% anual, es decir las empresas en la entidad enfrentarían un periodo de crisis y su participación en el mercado nacional perdería 0.8 puntos porcentuales, en 2012. En contraste, un aumento en la demanda externa, así como una política orientada a promover las exportaciones, tendría un mayor impacto en la recuperación de la industria de los alimentos con un crecimiento promedio de 5\% anual, entre 2009 a 2012, con un cambio positivo en su participación en el mercado nacional con 0.5 puntos porcentuales.

En el caso de la industria de autopartes, el escenario base muestra un crecimiento promedio de 3.8\% anual, de 2009 a 2012. Considerando un aumento en las importaciones de autopartes, es decir hasta un nivel que representen 10 puntos porcentuales sobre el valor del mercado nacional, en 2012. Se observa que el crecimiento de la producción en el periodo 2009 a 2012 sería de $0.36 \%$ anual, es decir prácticamente se estanca la producción en el estado de Guanajuato. Si bien es una industria relevante para el estado un aumento de la competencia del exterior podría generar que las empresas de la entidad pudieran perder hasta 3 puntos porcentuales del mercado nacional. Si actualmente cubre el 35\% del mercado nacional, un aumento de las importaciones llevaría a un nivel de $31 \%$, con una tendencia a la baja en la producción, lo cual ubicaría a las empresas en una situación de crisis. En efecto, el dato preliminar a 2010 indica que la industria esta enfrentando una mayor competencia acorde con el escenario alternativo 1 , de tal manera que la industria es muy sensible a las condiciones de la competencia externa.

Una promoción de las exportaciones, permitiría que la industria de autopartes repuntara en sus niveles de producción, a un ritmo de $4.7 \%$ anual en un punto porcentual por arriba del escenario base, y las empresas asentadas en la entidad podrían apropiarse de hasta un punto porcentual del mercado nacional. Los resultados muestran que la industria de autopartes es muy sensible a las condiciones del mercado internacional, si bien un aumento de la demanda extrema estimula la producción, es necesario desarrollar otros aspectos en las áreas de productividad, organización del trabajo, publicidad entre otras que ayuden a posicionar mejor las empresas de esta rama en el mercado nacional.

En el subsector de fabricación de productos de cuero y piel, la fabricación de calzado representa la principal actividad con una aportación al valor de la producción del orden de $72 \%$ para toda la entidad. La entidad contribuye con el $69.5 \%$ de la producción nacional. En el escenario base podría registrar un crecimiento de 4.9\% anual, entre 2010 a 2012 (Gráfica 7), de hecho en 2010 la participación de la entidad en el mercado nacional se mantiene estancada como 
Gráfica 6. Resultados para la industria de autopartes en Guanajuato

Autopartes: participación en el mercado nacional de la industria de Guanajuato. Escenario 1

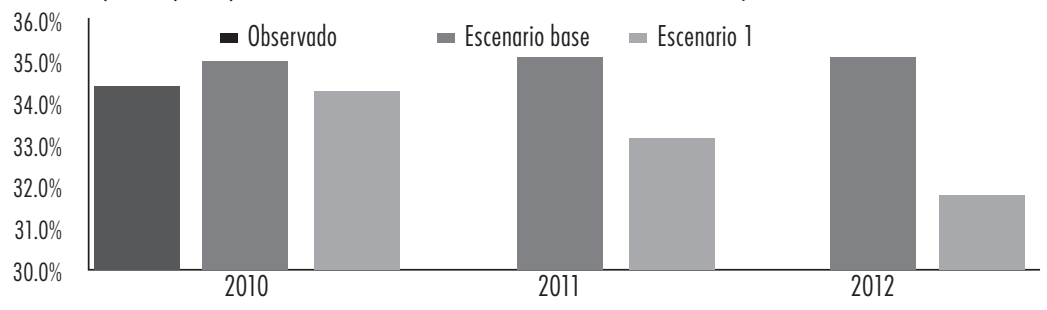

Autopartes: cambio en la participación del mercado a nivel nacional. Escenario 1 (puntos porcentuales)

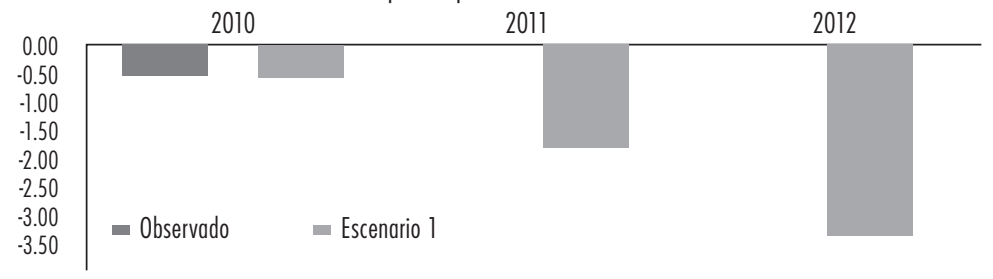

Autopartes: participación en el mercado nacional de la industria de Guanajuato. Escenario 2

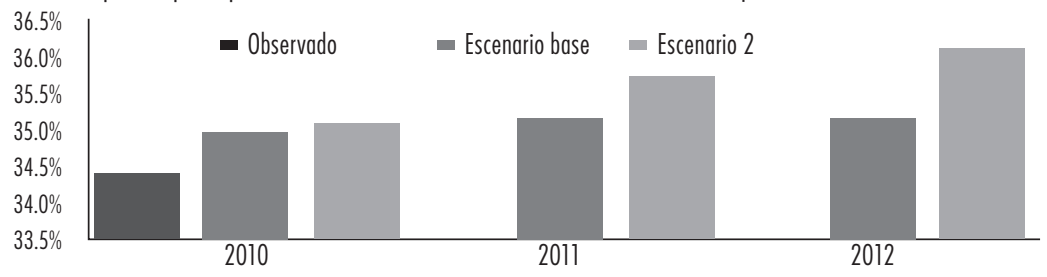

Autopartes: cambio en la participación del mercado a nivel nacional. Escenario 2 (puntos porcentuales)

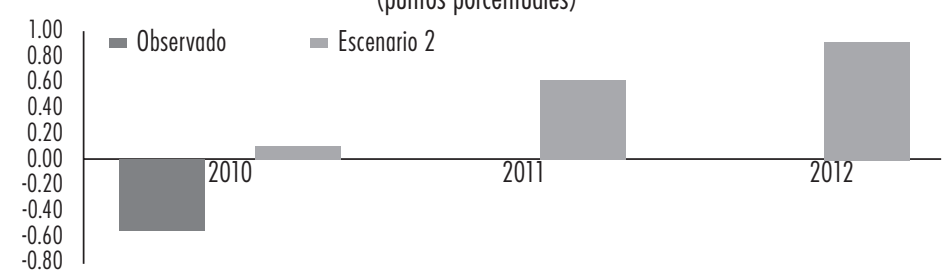

predice el modelo bajo el escenario base. Asumiendo un incremento en las importaciones, la industria del cuero y piel serían de las más afectadas, esto debido a que está sujeta a una mayor competencia internacional, y las importaciones 
Gráfica 7. Resultados para la industria de piel y calzado en Guanajuato

Piel y calzado: participación en el mercado nacional de la industria de Guanajuato. Escenario 1

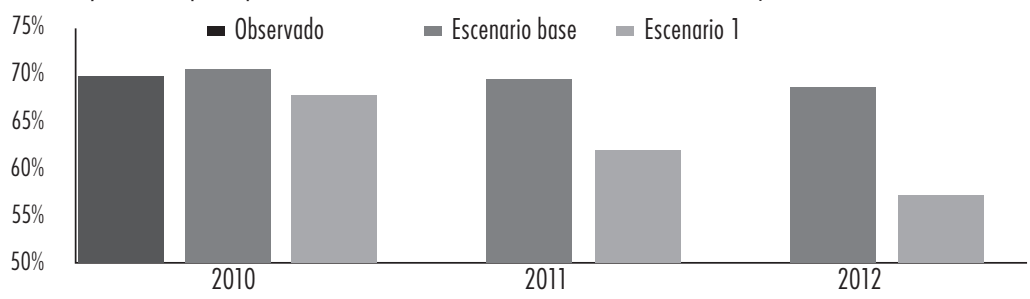

Piel y calzado: cambio en la participación del mercado a nivel nacional. Escenario 1 (puntos porcentuales)

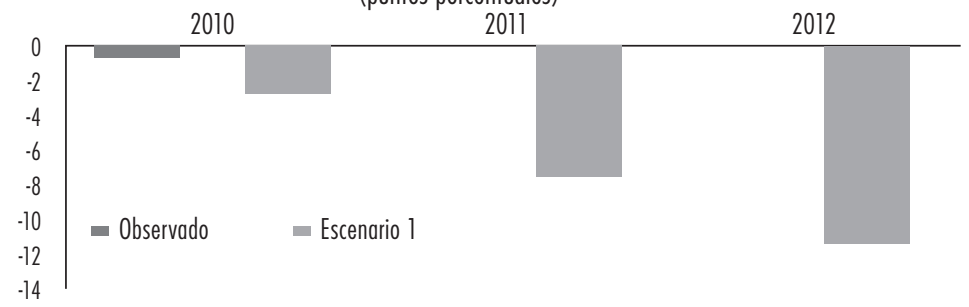

Piel y calzado: participación en el mercado nacional de la industria de Guanajuato. Escenario 2

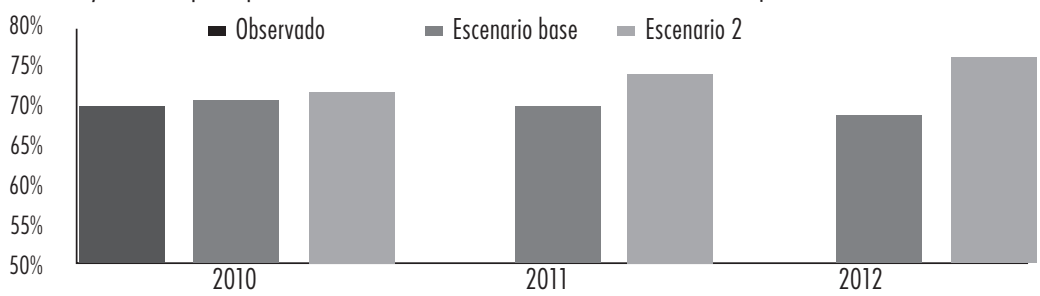

Piel y calzado: cambio en la participación del mercado a nivel nacional. Escenario 2 (puntos porcentuales)

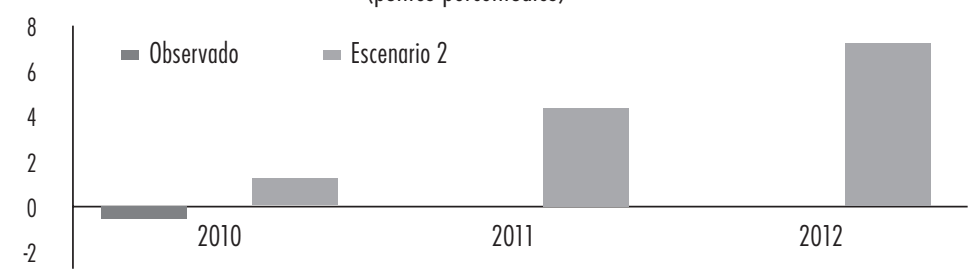

han crecido de manera acelerada. Así, por ejemplo, las empresas dedicadas a la producción de calzado en Guanajuato, asumiendo un aumento en las importaciones de 3\% anual, entre el año 2009 a 2012, implicaría que el valor de la 
producción registre una contracción del 1.3\% anual, eliminando la posible recuperación estimada bajo el escenario base (Gráfica 7). Esta situación implicaría una fuerte reducción en la participación del estado de Guanajuato en la producción nacional. En efecto se perderían, al 2012, prácticamente 11 puntos porcentuales del mercado nacional, es decir varias empresas de la entidad perderían presencia.

La fabricación de cuero, piel y de calzado sería de las ramas de las manufacturas de la entidad más beneficiadas de una promoción de exportaciones. Bajo el escenario alternativo, la producción crecería hasta una tasa de $8.5 \%$ promedio anual, de 2009 a 2012. Con este ritmo de crecimiento, y asumiendo que las importaciones no aumentan, las empresas de Guanajuato dedicadas a la fabricación de calzado podrían recuperar su posicionamiento en el mercado nacional y podrían incrementar su participación hasta en 7 puntos porcentuales (Gráfica 7).

\section{CONCLUSIONES}

La economía del Estado de Guanajuato muestra un gran dinamismo en las últimas dos décadas, con un crecimiento ligeramente superior al promedio nacional. Además, reportan con mayor frecuencia tasas de crecimiento positivas en comparación con el promedio nacional. Su contribución en la economía nacional se ha mantenido estable, muy cercana a un valor del $4 \%$. Se consolida como una de las entidades importantes en el centro del país. La industria de las manufacturas es un sector de gran importancia para la economía nacional ya que generó el 18\% del PIB, en el periodo de 2000 a 2008. En el caso del estado de Guanajuato, la industria manufacturera representó el $27.97 \%$ de su PIB estatal. Es el segundo sector más importante, después del sector servicios. Además, es una entidad que ha logrado una mayor especialización en la producción de manufacturas, aprovechando las condiciones de la apertura comercial y su ubicación geográfica.

Se considera que el Tratado de Libre Comercio ha permitido que la economía de Guanajuato muestre una mayor especialización en la industria de manufacturas. Los sectores analizados en el presente estudio: autopartes, alimentos y calzado, se orientan tanto al mercado interno, como al mercado externo, lo cual genera una mayor correlación con factores internos y externos.

La industria de equipo de autopartes se muestra como la predominante en las manufacturas, muy orientada al mercado externo. No obstante, muestra una tendencia descendente en su contribución a la economía. La industria de 
alimentos tiende a consolidarse como la tercera en importancia en el estado, muy orientada al mercado interno. La industria de productos de cuero no muestra dinamismo y su participación tiende estancarse, esto debido al acelerado incremento de las importaciones a nivel nacional.

La industria de manufacturas en Guanajuato tiene una estructura heterogénea, cuyas actividades están orientadas a distintos mercados. Así, por ejemplo, la industria de autopartes se concentra (72\%) en la fabricación de partes de sistemas de transmisión, con un gran dinamismo y una fuerte orientación al mercado externo, concentrada en los principales centros urbanos del estado. En la industria de alimentos destaca la producción de lácteos, conservas y la elaboración de pan y tortillas, así como la producción de alimentos para animales. Se orienta principalmente al mercado interno. La industria de cuero y piel, se concentra básicamente en la producción de calzado. De hecho, Guanajuato es el principal productor de calzado a nivel nacional, pero actualmente la rama muestra una tendencia descendente

La producción de calzado ha perdido presencia en el mercado nacional e internacional. El déficit comercial ha crecido desde el año 2002, debido a un acelerado crecimiento de las importaciones, a una tasa del 11\% anual, de 2000 a 2009 , indicando la gran sensibilidad de esta industria a las condiciones del mercado externo, así como las políticas comerciales.

Se genera un escenario base, asumiendo que el crecimiento de las importaciones se mantiene constante, estabilidad de precios y un crecimiento del pIB del 4\% anual para el periodo de 2010 a 2012. Los modelos econométricos estimados muestran que la industria de autopartes es bastante sensible a las condiciones del mercado externo. La industria de alimentos está muy vinculada al mercado interno. La industria del calzado desde principios de la presente década ha enfrentado una crisis derivada de un crecimiento acelerado de las importaciones, y se espera que mantenga esta tendencia negativa en los próximos años. Se definieron tres escenarios con fines de análisis, los datos preliminares a 2010 muestran que los modelos simulan adecuadamente la participación en el mercado nacional. Bajo el Escenario 1, que asume un aumento de las importaciones reduce el potencial de crecimiento en las tres industrias, de manera más grave en la de piel, cuero y calzado, en menor medida en la de autopartes y la de alimentos.

El Escenario 2, considera una expansión de la demanda externa y, en consecuencia, un aumento de las exportaciones, lo cual promueve el crecimiento de la industria de autopartes, pero la recuperación iniciaría hasta 2012. No obstante, es la industria con mayor potencial para incrementar su participación en el mercado nacional. En alimentos, no se observan impactos significativos en 
cuanto a su participación en el mercad nacional. Una promoción de exportaciones y un aumento de la demanda externa, la industria del cuero, piel y calzado responderían de manera inmediata y recobrarían un gran dinamismo, con un crecimiento promedio de $8.5 \%$ anual y una expansión hacia el mercado nacional de 7 puntos porcentuales

El crecimiento de la industria de manufacturas en Guanajuato depende de factores asociados al mercado interno como externo. Los modelos muestran que condiciones favorables en el mercado externo promoverían un mayor crecimiento en la autopartes y el calzado, pero este resultado considera que las empresas tienen una capacidad de respuesta para adaptarse a las nuevas condiciones y mejorar su posicionamiento en el mercado nacional. El posicionamiento en el mercado nacional también está asociado a otros factores como la competitividad, la publicidad, la regulación del mercado y el costo de abrir nuevas empresas, entre otros. Factores importantes para considerar en el diseño de una política industrial a nivel estatal.

\section{BIBLIOGRAFÍA}

Black, D. y V. Henderson, "A Theory of Urban Growth", Journal of Political Economy, vol. 107, núm. 21, 1999, pp. 252-284.

Clements, M. P. y D. F. Hendry, Forecasting Non-stationary Economic Time Sires, MIT Press, 1999.

Davis, D. R. y D. E. Weinstein, Economic Geography and Regional Production Structure: an Empirical Investigation, Federal Reserve Bank of New York, 1998, pp. 1-49.

Deaton, A. y J. Muellbauer, "An Almost Ideal Demand System", American Economic Review, 70(3), 1980, pp. 312-326.

Dickey, D. A. y W. A. Fuller, "Likelihood Ratio Statistics for Autoregressive Time Series with a Unit Root", Econometrica, 49(4), 1981, pp. 1057-1077.

Enders, W., "Applied Econometrics Time Series", Technometrics, 2d. Edition, 1995.

Engel, R. F. y C. W. J. Granger, "Cointegration and Error Correction: Representation, Estimation and Testing", Econometrica, 55, 1987, pp. 251-276. Fujita, M. y J. F. Thisse, Economics of Agglomeration, Cambridge University Press, 2002.

Gallup, J. L., J. D. Sachs, A. Mellinger, "Geography and Economic Development”, International Regional Science Review, 22, 1999, pp. 179-232. 
Granger, C. W. J. y P. Newbold, "Spurious Regressions in Econometrics". Journal of Econometrics 2, 1974, pp. 111-120.

Haldrup, N., "The Asymptotics of Single-Equation Cointegration Regressions with I(1) and I(2) Variables", Journal of Econometrics, 63, 1994, (1998), pp. 153-181.

Hanson, G. H., "Regional Adjustment to Trade Liberalization", Regional Science and Urban Economics, 28, 1998, pp. 419-444.

INEGI, Perspectiva Estadística, Guanajuato, 2009.

Johansen, S., "A Statistical Analysis of Cointegration for I(2) Variables", Econometric Theory, 11, 1995, pp. 25-59.

Johansen, S., "Statistical Analysis of Cointegration Vectors", Journal of Economic Dynamic and Control, 12, 1988, pp. 231-254

Juselius, K., The cointegrated VAR model. Methodology and Applications, Oxford University Press, 2006.

Krugman, P. R., "Increasing Returns and Economic Geography", Journal of Political Economy 99, 1991, pp. 483-499.

Kwiatkowsky, D., P. C. B., Phillips, P. Schmidt y Y. Shin, "Testing the Null Hypothesis of Stationary Against the Alternative of a Unit Root", Journal of Econometrics, 54, 1992, pp. 159-178

Maddala, G. y I. M. Kim Unit Roots, Cointegration, and Structural Change, Cambridge University Press, 1998.

Mendoza, J. E., "Manufacturing Specialization and Urban Aglommeration in the Largest Cities of Mexico", MPRA Paper No 2854, 2003.

Moomaw, R. L., "Productivity and City Size: a Critique of the Evidence", Quarterly Journal of Economics, vol. 95, 1981, pp. 675-688

Ng, S. y P. Perron, "Estimation and Inference in Nearly Unbalanced Nearly Cointegrated Systems", Journal of Econometrics, Elsevier, vol. 79(1), 1997, pp. 53-81.

Patterson, K., An Introduction to Applied Econometrics a Time Series Approach, St. Martin's Press, 2000.

Phillips, P. C. P. y P. Perron, "Testing for Unit Root in Time Series Regression", Biométrica, 75, 1988, pp. 335-346.

Rotemberg, J. y G. Saloner, "Competition and Human Capital Accumulation: a Theory of Interregional Specialization and Trade", Regional Science and Urban Economics, 30, 2000.

Sargent, J. y L. Matthews, “Combining Export Processing Zones and Regional Free Trade Agreements: Lesson from the Mexican Experience", World Development, 29(10), 2001, pp. 1739-1752

Tamura, R., "Regional Economies and Market Integration", Journal of Economic Dynamics and Control, 20, 1996, pp. 825-845. 


\section{ANEXO}

\section{Cuadro A1}

Pruebas de raíz unitaria

\begin{tabular}{|c|c|c|c|c|c|c|c|c|}
\hline \multirow{2}{*}{ Variable } & \multicolumn{3}{|c|}{ ADF } & \multirow{2}{*}{\multicolumn{2}{|c|}{ B }} & \multirow[b]{2}{*}{$C$} & \multirow{2}{*}{\multicolumn{2}{|c|}{$\eta$}} \\
\hline & $A$ & & C & & & & & \\
\hline \multicolumn{9}{|c|}{ Industria de alimentos } \\
\hline qalit & $-5.850^{\star}$ & $-5.782^{\star}$ & -0.282 & -3.972 & -4.0 & -0.278 & 0.071 & 0.077 \\
\hline$\Delta$ qalit & $-5.511^{\star}$ & $-5.549^{\star}$ & $-5.606^{\star}$ & -9.927 & -9.9 & $-10.06^{*}$ & 0.041 & 0.037 \\
\hline prt & -2.092 & 0.529 & 3.938 & -2.04 & & 5.104 & $0.941^{*}$ & $0.221^{\star}$ \\
\hline$\Delta$ prt & $-6.377^{\star}$ & $-6.320^{*}$ & $-4.764^{\star}$ & -5.985 & -5.9 & $-4.66^{*}$ & 0.134 & 0.084 \\
\hline $\mathrm{mt}$ & 0.046 & $-4.007^{*}$ & 0.759 & -4.159 & -3.8 & 1.151 & $0.549 *$ & $0.178^{\star}$ \\
\hline$\Delta \mathrm{mt}$ & $-6.839^{\star}$ & $-5.935^{\star}$ & $-5.903^{\star}$ & -14.71 & -11. & $-11.31^{*}$ & 0.304 & $0.225^{\star}$ \\
\hline$x t$ & $-4.955^{\star}$ & $-4.642^{*}$ & -0.099 & -3.543 & -3.4 & 0.759 & 0.307 & 0.038 \\
\hline$\Delta \mathrm{xt}$ & $-6.627^{\star}$ & $-6.721^{*}$ & $-6.588^{\star}$ & -8.220 & -8.3 & $-8.340^{\star}$ & 0.193 & $0.192^{\star}$ \\
\hline \multicolumn{9}{|c|}{ Industria de autopartes } \\
\hline qautt & $-3.781^{\star}$ & $-3.579^{\star}$ & -0.043 & -3.771 & -3.5 & -0.012 & 0.264 & $0.162^{\star}$ \\
\hline$\Delta$ quutt & $-5.867^{\star}$ & $-8.981^{\star}$ & $-9.063^{\star}$ & -18.71 & -16 . & $-16.77^{\star}$ & 0.335 & $0.299^{\star}$ \\
\hline prt & -1.301 & -1.312 & -0.331 & -1.42 & -1. & -0.398 & 0.135 & 0.140 \\
\hline$\Delta \mathrm{prt}$ & $-7.387^{\star}$ & $-7.378^{\star}$ & $-7.404^{\star}$ & -7.397 & -7.3 & $-7.404^{\star}$ & 0.159 & 0.138 \\
\hline $\mathrm{mt}$ & -3.294 & -1.171 & -0.068 & -3.29 & & 0.360 & $0.885^{\star}$ & 0.116 \\
\hline$\Delta \mathrm{mt}$ & $-9.171^{*}$ & $-9.255^{\star}$ & $-9.147^{\star}$ & -10.183 & -10. & $-9.464^{*}$ & 0.102 & 0.101 \\
\hline xt & -2.462 & -1.306 & 0.198 & -2.35 & -1. & 0.523 & $0.610^{*}$ & $0.200^{\star}$ \\
\hline$\Delta \mathrm{xt}$ & $-9.043^{\star}$ & $-9.070^{*}$ & $-9.076^{\star}$ & -9.230 & -9.2 & $-9.179^{\star}$ & 0.127 & 0.058 \\
\hline \multicolumn{9}{|c|}{ Industria de cuero y calzado } \\
\hline qpyct & $-6.109^{\star}$ & $-5.674^{\star}$ & 0.009 & $-6.052^{\star}$ & $-5.626^{*}$ & -0.267 & & 0.074 \\
\hline$\Delta$ qpyct & $-8.369 *$ & $-8.196^{\star}$ & $-8.109 *$ & $-30.86^{\star}$ & $-28.99 *$ & $-27.33^{\star}$ & & $0.500^{\star}$ \\
\hline prt & -2.722 & $-4.074^{\star}$ & 3.602 & -2.962 & $-5.774^{*}$ & 3.440 & & $0.238^{\star}$ \\
\hline$\Delta$ prt & $-9.671^{\star}$ & $-8.312^{*}$ & $-6.976^{\star}$ & $-9.728^{\star}$ & $-8.273^{*}$ & $-7.174^{*}$ & & 0.099 \\
\hline $\mathrm{mt}$ & $-6.091^{\star}$ & -2.652 & -0.290 & $-5.175^{\star}$ & $-4.636^{*}$ & 0.074 & & 0.132 \\
\hline$\Delta \mathrm{mt}$ & -9.748 & $-9.846^{\star}$ & $-9.939^{\star}$ & $-18.56^{\star}$ & $18.53^{*}$ & $-19.40^{\star}$ & & $0.359^{\star}$ \\
\hline xt & $-3.815^{\star}$ & $-3.798^{\star}$ & 0.047 & $-6.632^{\star}$ & $-6.666^{*}$ & 0.335 & & 0.073 \\
\hline$\Delta \mathrm{xt}$ & -13.917 & $-14.05^{\star}$ & $-14.15^{\star}$ & $-13.91^{\star}$ & $-14.05^{\star}$ & $-14.15^{*}$ & & 0.109 \\
\hline \multicolumn{9}{|c|}{ Variables macroeconómicas } \\
\hline $\mathrm{yt}^{\dagger}$ & -3.333 & $-3.260^{\star}$ & 0.720 & -3.204 & $-3.095^{\star}$ & 0.716 & & $0.194^{\star}$ \\
\hline$\Delta y^{\dagger}$ & $-10.473^{*}$ & $-10.49^{\star}$ & $-10.50^{\star}$ & $-10.95^{\star}$ & $-10.90^{\star}$ & $-10.64^{*}$ & & 0.069 \\
\hline
\end{tabular}

${ }^{\star}{ }^{\star}$ Rechazo de la hipótesis nula. Los valores críticos al 5\% para ADF y PP con T = 100 son: Modelo A $=-3.45$ (incluye constante y tendencia), Modelo $\mathrm{B}=-2.89$ (incluye constante) y Modelo $\mathrm{C}=-1.95$ (no incluye constante y tendencia) (Maddala y Kim, 1998), p. 64. Los valores críticos de la prueba KPSS a un nivel del $5 \%$ de significancia son: $\eta_{\mu}=0.463$ (incluye constante) y $\eta_{\tau}=0.146$ (incluye constante y tendencia). (Kwiatkowski, et al.,1992). Periodo: 2005(1)-2009(12) 